C. Aerts, T.R. Bedding, \& J. Christensen-Dalsgaard, eds.

\title{
Nonlinear Convective Pulsation Models of Second Overtone SMC Cepheids
}

\author{
M. Marconi \\ Osservatorio Astronomico di Capodimonte, Via Moiariello 16, 80131 \\ Napoli, Italy, e-mail: marcella@na.astro.it \\ G. Bono, F. Caputo \\ Osservatorio Astronomico di Roma, Via Frascati 33, 00040 Monte \\ Porzio Catone, Italy
}

\begin{abstract}
Nonlinear convective pulsation models representative of SMC Cepheids pulsating in the second overtone mode are presented. Model predictions are compared with observations and implications are derived for second overtone selection criteria. The role of overtone Cepheids as distance indicators is finally investigated and the derived distance scale is at odds with the so called short distance scale.
\end{abstract}

\section{Introduction}

The occurrence of first overtone (FO) pulsators among Classical Cepheids was originally suggested by Pel \& Lub (1978), but it was definitively settled by the large sample of variable stars collected by the microlensing experiments (MACHO, EROS, OGLE). These teams were also able to detect some tens of doublemode Magellanic Cepheids pulsating simultaneously in the first and in the second overtone (Beaulieu et al., 1997; Alcock et al., 1999; Udalski et al., 1999a, U99a). Moreover, Udalski et al. (1999b, U99b) discovered a dozen low-amplitude, shortperiod second overtones (SOs) in a sample of 2300 SMC Cepheids. We selected this sample to test the predictive capability of our nonlinear convective SO models for relatively metal poor Cepheids.

\section{The models}

The full amplitude, nonlinear convective models presented in this investigation were constructed by adopting the input physics and physical assumptions discussed in Bono et al. (1999, BMS), Bono et al. (2000) and Bono et al. (2001, BCM01). To cover the observed period range of SO Cepheids we constructed four different sequences of models at fixed chemical composition $Y=0.25, Z=0.004$ and stellar masses equal to $3.25,3.5,3.8$ and $4.0 M_{\odot}$. The luminosity of these models was fixed according to the mass-luminosity (ML) relation adopted by BMS. To assess how the topology of the instability strip changes when moving toward lower luminosities, the previous models were implemented with three series of FOs with stellar masses equal to 5.0, 5.5 and $5.8 M_{\odot}$. 


\section{Comparison between theory and observations}

In Fig. 1 the Wesenheit index of pure SO pulsators detected by U99b is compared with the index based on SO models, for an assumed distance modulus $\mu_{0}=19.1$ mag. Apart from a few objects which appear to be systematically brighter/cooler than predicted, the agreement is quite satisfactory. Since we suspected that some variables classified as FO pulsators by Udalski et al. could instead be pure $\mathrm{SOs}$, we included in the SO sample all the variables within $3 \sigma(\sigma=0.03 \mathrm{mag})$ from the predicted SO loci and we found that the color distribution of the new candidates is similar to the V-I distribution of FO/SO double mode Cepheids. The agreement between the theoretical and the empirical Wesenheit functions for both FO and SO pulsators supports the preductive capability of present models. However, before driving any definite conclusion, we also need to explore the behavior of amplitudes and Fourier parameters. The observed $R_{21}$ values are too uncertain and the comparison with theory is useless. As for the $\Phi_{21}$ parameter, the agreement between empirical values and theoretical predictions is very good but it appears that SO and FO Cepheids cover the same range of values, preventing the use of this parameter to single out $\mathrm{SO}$ pulsators. As for pulsation amplitudes, theory seems to overestimate amplitudes, at least in the short period range, for both SO and FO pulsators but amplitude could be a useful quantity to discriminate the pulsation mode.

\section{Overtone Cepheids as distance indicators}

To disclose whether SOs are good standard candles we derived, on the basis of current SO models, the following Period-Luminosity-Color (PLC) relation:

$$
M_{V}=3.961( \pm 0.005)-3.905( \pm 0.01) \log P+3.250( \pm 0.054)(V-I) \text {, }
$$

where $M_{V}$ and $(V-I)$ are intensity-weighted mean values, $P$ is the period (d), and the standard deviation is 0.004 . The spread of this relation is small because the temperature width of the SO instability strip is substantially smaller than F and FO strips. Interestingly enough, we find that by fitting the sample of pure SO pulsators, together with the FO/SO Cepheids, the SMC distance modulus is $19.11 \pm 0.08 \mathrm{mag}$, for an assumed mean reddening value $E(B-V)=$ $0.054 \pm 0.029$. The current SMC distance estimate supports the distance modulus determination derived by Bono et al. $(1999, D M=19.19 \pm 0.17 \mathrm{mag})$ on the basis of the fundamental PLC $(V, B-V)$ relation and data available in the literature, as well as several empirical results for the SMC distance (Laney \& Stobie, 1994; Kovacs, 2000; Groenewegen, 2000). Given the above result, we decided to estimate the SMC distance by adopting the theoretical PLC relation for FOs. By taking into account current and old (Bono et al., 1999) models, we found the following $\mathrm{PLC}(V, V-I)$ relation:

$$
M_{V}=3.61( \pm 0.03)-3.85( \pm 0.02) \log P+3.33( \pm 0.09)(V-I)
$$

with a standard deviation of $0.03 \mathrm{mag}$. The fit of this relation to both pure FOs and FO/SO Cepheids supplies $D M=19.16 \pm 0.19 \mathrm{mag}$, for the same mean 


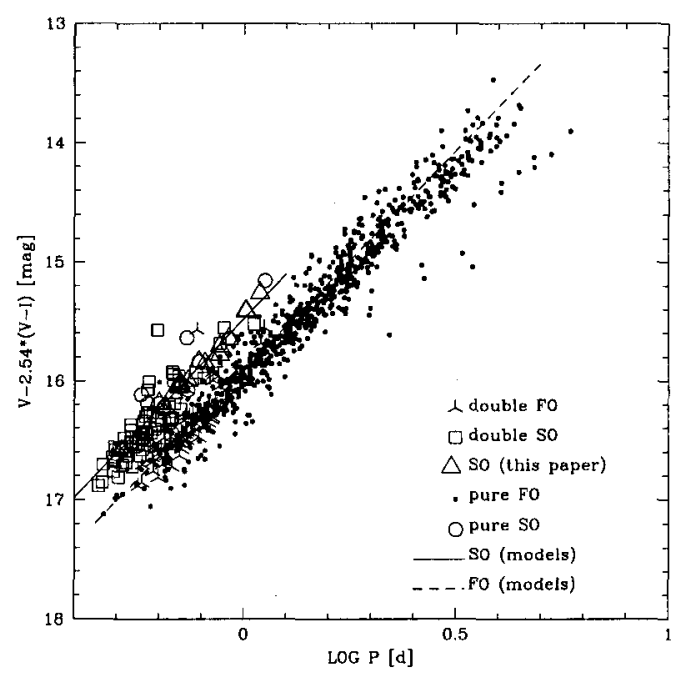

Figure 1. Wesenheit index versus period for pure SO pulsators (open circles, U99b), SO component in FO/SO Cepheids (open squares, U99a), pure FO pulsators (filled circles, U99b), FO component in FO/SO Cepheids (filled squares, U99a). Open triangles show the new selected SO pulsators, while solid and dashed lines display the predicted Wesenheit indices for second and first overtones respectively. Data were plotted by assuming $D M=19.1$ mag.

reddening adopted for SO variables. Even though FOs cover different period and luminosity ranges, the new distance agrees, within the errors, with the distance based on SOs. At the same time, both of them are at odds with SMC distances based on red clump stars $(D M=18.63 \pm 0.07 \mathrm{mag})$ and on field RR Lyrae stars $(D M=18.66 \pm 0.16 \mathrm{mag})$ derived by Udalski (1998) and by Udalski et al. (1998).

But what does this distance scale imply for the LMC distance scale? By adopting the PLC relation based on FO models for $Z=0.008$, namely

$$
M_{V}=3.60( \pm 0.02)-3.82( \pm 0.02) \log P+3.26( \pm 0.06)(V-I)
$$

with a standard deviation of $0.02 \mathrm{mag}$, and the database provided by Udalski et al. (1999c) for both pure FO and FO components of double mode LMC pulsators, we derive $D M=18.62 \pm 0.18 \mathrm{mag}$. This result confirms that the distance scale based on current models is at odds with the extremely short distance scale (e.g., Gould \& Uza, 1998; Udalski et al., 1998). 


\section{References}

Alcock, C., et al. 1999, AJ, 117, 920

Beaulieu, J.P., et al. 1997, A\&A, 318, L47

Bono, G., Caputo, F., Castellani, V., \& Marconi, M. 1999, ApJ, 512, 711

Bono, G., Caputo, F., \& Marconi, M. 2001, MmRAS, 325, 1353 (BCM01)

Bono, G., Castellani, V., \& Marconi, M. 2000, ApJ, 532, L129

Bono, G., Marconi, M., \& Stellingwerf, R.F. 1999, ApJS, 122, 167 (BMS)

Gould, A., Uza, O. 1998, ApJ, 494, 118

Kovacs, G. 2000, A\&A, 360, L1

Laney, C.D. \& Stobie, R.S. 1994, MmRAS, 266, 441

Pel, J.W., Lub, J. 1978 in The HR diagram - The 100th anniversary of H.N. Russell, (Reidel, Dordrecht), 229

Udalski, A. 1998, AcA, 48, 113

Udalski, A., Pietrzynski, G., Wozniak, P., Szymanski, M., Kubiak, M., \& Zebrun, K. 1998, ApJ, 509, L25

Udalski, A., et al. 1999a, AcA, 49, 1 (U99a)

Udalski, A., et al. 1999b, AcA, 49, 45 (U99b)

Udalski, A., et al. 1999c, AcA, 49, 223 (U99c)

\section{Discussion}

D. Welch : Did I understand you correctly when you said that you reclassified the claimed modes by using the Wesenheit Function? Observationally, I have found the Fourier parameters for SO (singly-periodic) Cepheids to be very distinct from FOs in the MACHO SMC data.

M. Marconi : We used the comparison in the Wesenheit plane to select pure SO Cepheids in the OGLE SMC sample and the resulting SO data are very similar to the FOs in the $\Phi_{21}$ versus period plot. Perhaps this is due to a worse $\mathrm{S} / \mathrm{N}$ ratio than for your data. 